\title{
Clima e sobreposição da distribuição de Aedes aegypti e Aedes albopictus na infestação do Estado de São Paulo
}

Climate and the superimposed distribution of Aedes aegypti and Aedes albopictus on infestation of São Paulo State, Brazil

\author{
Carmen Moreno Glasser ${ }^{\mathrm{a}}$ e Almério de Castro Gomes ${ }^{\mathrm{b}}$ \\ a Diretoria de Combate a Vetores da Superintendência de Controle de Endemias. São Paulo, SP, Brasil. \\ ${ }^{b}$ Departamento de Epidemiologia da Faculdade de Saúde Pública da Universidade de São Paulo. São \\ Paulo, SP, Brasil
}

\section{Descritores}

Aedes. Distribuição espacial. Insetos vetores. Clima. Ecologia de vetores. Fatores meteorológicos. Aedes albopictus. Aedes aegypti.

\section{Keywords}

Aedes. Residence characteristics. Vector insects. Climate. Ecology, vectors. Meteorological factors. Aedes albopictus. Aedes aegypti.

\section{Resumo}

Objetivo

Estudar a influência de fatores climáticos e da sobreposição da distribuição das populações de Aedes aegypti e de Aedes albopictus na expansão geográfica dessas espécies no Estado de São Paulo.

\section{Métodos}

Foram obtidas informações de órgãos do governo sobre ocorrência de focos de Ae. aegypti e de Ae. albopictus e sobre o estabelecimento dessas espécies em municípios do Estado de São Paulo ano a ano - entre 1985 e 1995 -, além de informações referentes a temperatura e índices pluviométricos. Dois indicadores foram utilizados: infestação e velocidade.

Resultados e Conclusões

Verificou-se que quanto menor a temperatura, mais lento foi o processo de expansão geográfica da população de Ae. aegypti. Esse fator teve influência preponderante na determinação dos diversos padrões macrorregionais de expansão geográfica dessa espécie no Estado de São Paulo. Não foram encontradas indicações claras sobre a influência da temperatura na dispersão de Ae. albopictus. A influência dos índices pluviométricos nos padrões de expansão geográfica dessas espécies somente foi constatada para Ae aegypti numa das áreas do Estado. Não se verificou influência da presença anterior de Ae. albopictus para o estabelecimento de Ae. aegypti.

\section{Abstract}

\section{Objective}

To study the influence of climatic factors and superimposed distribution of Aedes aegypti and Aedes albopictus populations on their geographic dispersal in the state of São Paulo.

\section{Methods}

Data from government agencies on Ae. aegypti and Ae. albopictus foci and their distribution throughout counties of the state of São Paulo year by year, from 1985 to 1995, were obtained. Temperatures and rainfall data were also collected. Two indicators were used: infestation and rate.

\section{Results and Conclusions}

The analysis indicated that the lower the temperature, the slower the geographic

Baseado na dissertação de mestrado apresentada à Faculdade de Saúde Publicada Universidade de São Paulo, 1997. Edição subvencionada pela Fundação de Amparo a Pesquisa do Estado de São Paulo (Fapesp - Processo n. 01/01661-3). Recebido em 24/5/2001. Reapresentado em 19/11/2001. Aprovado em 18/12/2001.
Carmen Moreno Glasser

Superintendência de Controle de Endemias (Sucen)

Rua Paula Souza, 166

01027-000, São Paulo, SP, Brasil

E-mail: carmen@sucen.sp.gov.br 
dispersal of Ae. aegypti population. This factor predominantly influenced the macro regional dispersal patterns of the species in the state of São Paulo. There was no clear influence of temperature on the dispersal of Ae. albopictus. The influence of rainfall on dispersal patterns was seen only for Ae. aegypti in a state area. No influence was detected on the prior presence of Ae. albopictus on the establishment of Ae. aegypti.

\section{INTRODUÇÃO}

Múltiplos são os fatores envolvidos na dispersão de culicídeos domiciliados. A expansão geográfica de populações de Aedes (Stegomyia) aegypti e Aedes (Stegomyia) albopictus sofre influência de fatores ambientais e sociais, entre os quais o clima, a densidade demográfica e a atividade econômica. Em relato recente, ${ }^{6}$ foi caracterizado o processo de infestação das várias regiões do Estado de São Paulo diferenciadas quanto aos dois últimos fatores. Observou-se que, na macrorregião com maior densidade demográfica e importância econômica, a velocidade de expansão geográfica de Ae. aegypti foi menor, ao contrário de observações em muitos outros países. ${ }^{8}{ }^{11}$ Entretanto, quando se analisou o comportamento dessa espécie dentro de cada macrorregião, observou-se que o processo de infestação deu-se mais rapidamente nas áreas com maior densidade demográfica, e os municípios mais populosos foram geralmente os primeiros a ser infestados. Embora também tenham sido identificados diferentes padrões macrorregionais na expansão geográfica de Ae. albopictus, estes pareceram estar principalmente relacionados à diferenciação das atividades de vigilância e controle dessa espécie nessas macrorregiões. ${ }^{6}$

A influência do clima na distribuição e abundância dos insetos e na epidemiologia das doenças por eles veiculadas é bastante conhecida. Ae. aegypti tem ampla distribuição na região tropical e subtropical limitada por isoterma do mês mais frio de $10^{\circ} \mathrm{C}$, enquanto Ae. albopictus é uma espécie adaptada ao frio da Ásia setentrional, existindo registros de sua presença em cidades com temperatura média de até $-4,8^{\circ} \mathrm{C}$ no mês mais frio. ${ }^{14,15}$ São cada vez maiores as evidências de que a ampliação das áreas de ocorrência de doenças transmitidas por vetores em vários continentes e seu agravamento tiveram, entre outros determinantes, o aumento da temperatura do planeta, especialmente nos últimos 100 anos $\left(0,8^{\circ} \mathrm{C}\right.$ na Europa; $0,3^{\circ} \mathrm{C}$ a $0,8^{\circ} \mathrm{C}$ na Ásia).$^{7}$ Outro fator climático importante na abundância de $A e$. aegypti e Ae. albopictus é a precipitação pluviométrica, a qual influi principalmente na densidade de criadouros devido ao aumento de recipientes artifi- ciais e naturais com acúmulo de água no extradomicílio, nos períodos e locais com mais freqüência e intensidade de chuva. ${ }^{8}$

Tem sido bastante discutida a possibilidade de a redução de abundância das populações de Ae. aegypti ou de Ae. albopictus, quando da sobreposição de sua distribuição em diferentes regiões do mundo, ser resultado de competição interespecífica. Em algumas áreas dos Estado Unidos, verificou-se redução de abundância da população de $A e$. aegypti quando $A e$. albopictus nelas se estabeleceu. ${ }^{1}$ Em áreas infestadas por Ae. albopictus no Sudeste Asiático, quando houve introdução e expansão geográfica de Ae. aegypti, verificou-se o contrário. ${ }^{3}$ Vários autores têm realizado estudos sobre o assunto sem conseguir confirmar se esses fenômenos foram realmente resultantes de competição entre essas espécies. ${ }^{10,15}$

Dessa forma, considerando a importância das condições climáticas na dispersão das populações de $A e$. aegypti e Ae. albopictus e a possível competição entre populações desses culicídeos merecer ser investigada, o presente trabalho tem como objetivo avaliar a influência da temperatura, dos índices pluviométricos e da sobreposição da distribuição dessas espécies nos padrões de expansão geográfica de suas populações no Estado de São Paulo.

\section{MÉTODOS}

Os conceitos utilizados sobre ocorrência de foco, estabelecimento e expansão geográfica de cada espécie foram os mesmos empregados em trabalho anterior. ${ }^{6}$ Foram obtidas informações sobre ocorrência de focos de Aedes aegypti e de Ae. albopictus durante o período de 1985 a 1995 , definindo-se o município como unidade geográfica, e o ano, como unidade de tempo. As informações foram obtidas dos seguintes órgãos:

- Superintendência de Controle de Endemias: informações anuais* sobre ocorrência de focos de cada espécie em municípios anteriormente livres de infestação e atualmente infestados;

- Instituto Agronômico - Seção de Climatologia Agrícola: mapas do Estado com isotermas ${ }^{16} \mathrm{e}$ dados anuais de temperatura média de julho e milí- 
metros de chuva das estações meteorológicas;

- Departamento de Águas e Energia Elétrica-Centro Tecnológico de Hidráulica e Recursos Hídricos: mapa do Estado com isoietas. ${ }^{4}$

As duas cartas climáticas básicas com isotermas e isoietas (Figuras 1 e 2) referem-se a elementos macroclimáticos e foram elaboradas a partir de séries históricas de dados coletados em estações meteorológicas e de outras informações adicionais. ${ }^{4,16}$ Deu-se preferência às isotermas de julho por ser este o mês mais frio e, portanto, aquele mais desfavorável ao estabelecimento dos dois cilicídeos. Procedeu-se à comparação da temperatura média de julho e dos milímetros de chuva anuais das diversas estações meteorológicas do Estado (médias para o período de 1985 a 1995), com as faixas de temperatura e de milímetros de chuva correspondentes à localização de cada estação nas cartas climáticas, verificando-se que as mesmas representam de forma apropriada as condições de temperatura e chuva do período de estudo.

Foram utilizados dois indicadores criados em trabalho anterior: ${ }^{6}$

- indicador infestação: porcentagem de municípios com estabelecimento da espécie em relação aos que apresentaram focos dessa mesma espécie;

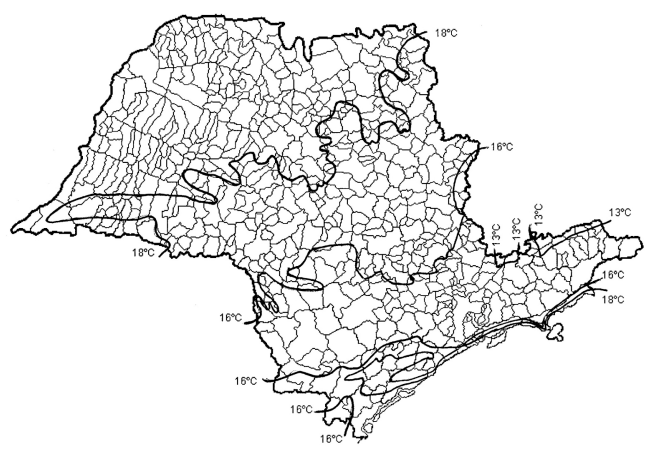

Figura 1 - Isotermas de julho. Estado de São Paulo (elaborado com dados até 1974).

Fonte: Instituto Agronômico. Secretaria de Agricultura.

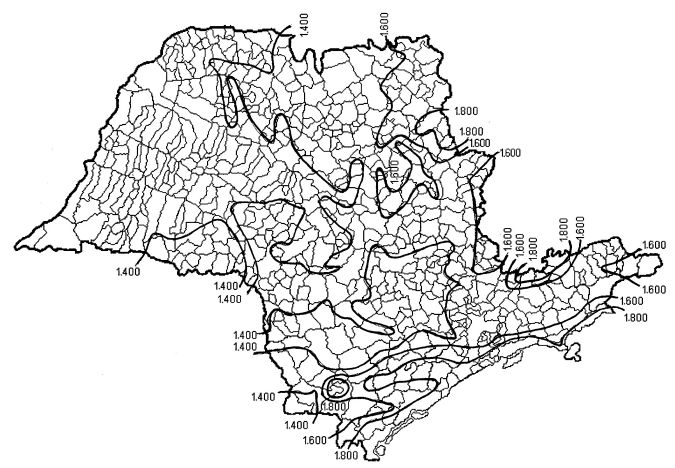

Figura 2 - Isoietas anuais. Estado de São Paulo, 1974 a 1988. Fonte: Departamento de Águas e Energia Elétrica. Secretaria de Recursos Hídricos, Saneamento e Obras.
- indicador velocidade: número de anos decorridos para infestar $10 \%, 50 \%$ e $90 \%$ dos municípios, após estabelecimento da espécie em municípios pertencentes a cada área de estudo.

A análise do indicador infestação foi realizada para os municípios com focos de Ae aegypti e Ae albopictus, segundo: áreas delimitadas por isotermas de julho (Figura 1), descartada aquela com isotermas de julho inferiores a $13^{\circ} \mathrm{C}$, por incluir apenas a sede do município de Campos do Jordão, SP; áreas delimitadas por isoietas anuais (Figura 2) e conjunto de municípios infestados e o de não infestados anteriormente pela outra espécie. Essa análise consistiu em estudo de associação entre as variáveis isoterma em três categorias, isoieta em quatro categorias e infestação anterior pela outra espécie, nas modalidades sim e não, com a variável estabelecimento da espécie, nas modalidades sim ou não. Para avaliar o grau de associação, determinaram-se os coeficientes de Yule para tabelas $2 \times 2$ e de Cramér para tabelas $2 \times 3$ e $2 \times 4$. Esses coeficientes variam de 0 a 1 , sendo que o 0 indica haver independência entre as variáveis, e o 1 , associação perfeita. ${ }^{2}$

Em trabalho anterior, verificou-se que $75 \%$ dos municípios em que se registrou o estabelecimento de Ae. aegypti ou Ae. albopictus distavam até $34 \mathrm{~km}$ e $60 \mathrm{~km}$, respectivamente, do município mais próximo infestado. ${ }^{6}$ Para minimizar a influência da distância, foram excluídos da análise os municípios que, quando apresentaram focos de Ae. aegypti, distavam $34 \mathrm{~km}$ ou mais do município infestado mais próximo, e, para Ae. albopictus, aqueles que distavam $60 \mathrm{~km}$ ou mais.

Para descartar a interferência das diferentes formas de atuação do programa de controle sobre $A e$. albopictus ${ }^{6}$ restringiu-se a análise da influência da temperatura e dos índices pluviométricos para $A e$. albopictus aos municípios sem infestação anterior por Ae. aegypti, ou seja, àqueles nos quais as ações de vigilância e controle de Ae. albopictus foram iguais às executadas para Ae. aegypti.

A determinação do indicador velocidade somente foi possível para áreas delimitadas por isotermas de julho devido à descontinuidade geográfica verificada nas áreas referentes às outras duas variáveis. Compararam-se apenas as três grandes áreas delimitadas no Estado quanto à temperatura, excluindo-se o litoral devido à descontinuidade geográfica das áreas delimitadas nessa região com as correspondentes no restante do Estado (Figura 1) e por abranger reduzido número de municípios, impossibilitando efetuar a análise em separado. 


\section{RESULTADOS}

\section{Expansão geográfica de $A e$. Aegypti}

\section{Influência da temperatura}

Verificou-se forte associação entre a temperatura média de julho e o estabelecimento de Ae. aegypti (Tabela 1$)(-p<0,001 ;$ coeficiente de Cramér $=0,8309)$. O percentual de municípios onde a espécie se estabeleceu foi tanto maior quanto mais elevada a faixa de temperatura da área em que eles se localizavam. Verificou-se também que a velocidade de ocupação de novos municípios foi tanto maior quanto maior a faixa da temperatura (Figura 3): na área onde esta foi maior que $18^{\circ} \mathrm{C}$, em menos de dois anos após o estabelecimento da espécie em algum de seus municípios, $10 \%$ destes estavam infestados, subindo para $50 \%$ e $90 \%$ após três e cinco anos, respectivamente; a área com temperatura de $16^{\circ} \mathrm{C}$ a $18^{\circ} \mathrm{C}$ somente apresentou $10 \%$ e $50 \%$ de seus municípios infestados, respectivamente, após três e seis anos, e, na área com temperatura de $13^{\circ} \mathrm{C}$ a $16^{\circ} \mathrm{C}$, menos de $10 \%$ de seus municípios encontravam-se infestados após quatro anos.

\section{Influência dos índices pluviométricos}

Para minimizar a interferência da temperatura na análise da influência da chuva na expansão geográfica da população do culicídeo, realizou-se separadamente investigação para cada área segundo faixa

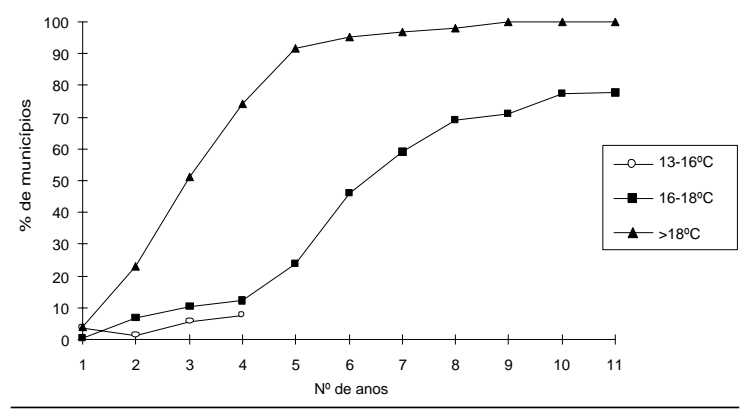

Figura 3 - Percentual de municípios infestados por Ae. aegypti em diferentes áreas quanto à faixa de temperatura média de julho, segundo número de anos transcorridos após o estabelecimento da espécie em cada área. Estado de São Paulo, 1985 a 1995 de temperatura. Porém, devido ao pequeno número de municípios que puderam ser selecionados para a área na faixa de temperatura de $13^{\circ} \mathrm{C}$ a $16^{\circ} \mathrm{C}$, somente foi possível realizar o estudo estatístico de associação para os municípios pertencentes às outras duas áreas. Mesmo nestas, foi necessário agrupar os municípios em apenas duas faixas: com menos de 1.400 $\mathrm{mm}$ e com mais de $1.400 \mathrm{~mm}$ de chuva anual. Somente verificou-se fraca associação entre índices pluviométricos e estabelecimento da espécie dentre os municípios na área com temperatura de $16^{\circ} \mathrm{C}$ a $18^{\circ} \mathrm{C}(\mathrm{p}<0,05$; coeficiente de Yule $=0,381)$; para a faixa de menos de $1.400 \mathrm{~mm}$ de chuva, a espécie estabeleceu-se em 56,3\% dos municípios com foco, e, para a faixa de mais de $1.400 \mathrm{~mm}$, esse percentual subiu para $74,2 \%$.

\section{Influência da infestação anterior por Aedes albopictus}

Até 1995, foi registrada a ocorrência de focos de Ae. aegypti em 440 municípios, já excluídos aqueles que distavam $34 \mathrm{~km}$ ou mais do município infestado mais próximo. Desse total, apenas 33 municípios já estavam infestados por Ae. albopictus e 407 não, verificando-se estabelecimento de $A$ e. aegypti, respectivamente, em $66,7 \%$ e $68,8 \%$ desses municípios, resultado que indicou não existir associação entre infestação anterior por Ae. albopictus e estabelecimento de Ae. aegypti ( $>00,5)$.

\section{Expansão geográfica de Ae. Albopictus}

\section{Influência da temperatura}

A Tabela 2 mostra que o percentual de municípios com estabelecimento de Ae. albopictus foi muito semelhante para os municípios pertencentes às duas primeiras faixas de temperatura, aumentando para aqueles com temperatura maior que $18^{\circ} \mathrm{C}$. No entanto, não se verificou associação significativa entre as duas variáveis $(\mathrm{p}>0,05)$.

A Figura 4 mostra que a velocidade de ocupação dos municípios foi diferente em cada uma das três áreas quanto à temperatura. $\mathrm{Na}$ área com temperatura na fai-

Tabela 1 - Municípios com ocorrência de focos de Ae. aegypti situados até $33 \mathrm{~km}$ de área infestada, segundo temperatura média de julho e estabelecimento da espécie. Estado de São Paulo, 1985-1995.

\begin{tabular}{|c|c|c|c|c|c|c|}
\hline \multirow[t]{2}{*}{$\begin{array}{l}\text { Temperatura } \\
\text { média }\end{array}$} & \multicolumn{2}{|c|}{$\begin{array}{c}\text { Municípios sem estabelecimento } \\
\text { da espécie }\end{array}$} & \multicolumn{2}{|c|}{$\begin{array}{c}\text { Municípios com estabelecimento } \\
\text { da espécie }\end{array}$} & \multicolumn{2}{|c|}{ Total } \\
\hline & $\mathrm{N}$ & $\%$ & $\mathrm{~N}$ & $\%$ & $\mathrm{~N}$ & $\%$ \\
\hline 13 a $16^{\circ} \mathrm{C}$ & 16 & 66,7 & 08 & 33,3 & 24 & 100,0 \\
\hline 16 a $18^{\circ} \mathrm{C}$ & 70 & 33,0 & 142 & 67,0 & 212 & 100,0 \\
\hline$>18^{\circ} \mathrm{C}$ & 52 & 25,5 & 152 & 74,5 & 204 & 100,0 \\
\hline Total & 138 & 31,4 & 302 & 68,6 & 440 & 100,0 \\
\hline
\end{tabular}


xa de $13^{\circ} \mathrm{C}$ a $16^{\circ} \mathrm{C}$, Ae. albopictus chegou a infestar $10 \%$ dos municípios dois anos após seu estabelecimento em algum município da área, 50\% após sete anos e não atingiu $90 \%$ após nove anos. A área com temperatura de $16^{\circ} \mathrm{C}$ a $18^{\circ} \mathrm{C}$ apresentou $10 \%$ e $50 \%$ de seus municípios infestados, respectivamente, após três e seis anos, e 90\%, no oitavo ano. No entanto, sua velocidade de expansão geográfica foi menor na área com temperatura acima de $18^{\circ} \mathrm{C}$, com apenas $43,7 \%$ dos municípios infestados após oito anos.

\section{Influência dos índices pluviométricos}

Como não se verificou associação entre temperatura e percentual de municípios com estabelecimento de Ae. albopictus, o estudo da influência da chuva não foi realizado separadamente para cada área quanto à temperatura. Os percentuais de municípios com estabelecimento de Ae. albopictus, para as várias faixas de índices pluviométricos, foram semelhantes, não se verificando associação entre as duas variáveis ( $\mathrm{p}>0,10$ ): $50 \%$ para a faixa de menos de $1.400 \mathrm{~mm}$ de chuva anual, $48,0 \%$ para a de $1.400 \mathrm{~mm}$ a $1.600 \mathrm{~mm}$, $40,6 \%$ para a de $1.600 \mathrm{~mm}$ a $1.800 \mathrm{~mm}$ e $52,0 \%$ para a de mais de $1.800 \mathrm{~mm}$.

\section{Influência da infestação anterior por Ae. aegypti}

Até 1995, foi registrada a ocorrência de focos de Ae. albopictus em 617 municípios, já excluídos aqueles que distavam $60 \mathrm{~km}$ ou mais do município infestado mais próximo. Desse total, 171 municípios já estavam infestados por Ae. aegypti e 446 não, verificando-se o estabelecimento de Ae. Albopictus, respectivamente, em $88,9 \%$ e em $47,1 \%$ desses municípios; ou seja, o percentual de municípios com estabelecimento de Ae. albopictus foi muito maior dentre os municípios com infestação anterior por $A e$. aegypti $(\mathrm{p}<0,0001$; coeficiente de Yule $=0,7998)$.

\section{DISCUSSÃO}

A temperatura tem influência direta na distribuição geográfica de populações vetoras de doenças, permitindo estabelecer limites para ocorrência de espécies como Ae. aegypti e Ae. albopictus. Nawrocki \& Hawley ${ }^{12}$ estimaram o limite ao norte, nos Estados Unidos, da distribuição futura de Ae. albopictus com base em dados da distribuição da espécie na Ásia setentrional: isoterma de $0^{\circ} \mathrm{C}$ do mês mais frio para a espécie ultrapassar o inverno, estabelecendo-se no local, e de $-5^{\circ} \mathrm{C}$ para sua expansão durante o verão. Esse limite estimado fica muito mais ao norte do que aquele relatado para a distribuição de Ae. aegypti nesse país. ${ }^{12,15}$ Além disso, geralmente este não é encontrado acima de 1.000 metros, apesar de ter sido observada sua presença em altitudes acima de 2.000 metros, em locais cuja temperatura média anual é de $17^{\circ} \mathrm{C} .{ }^{13}$

O Estado de São Paulo localiza-se entre $20,3^{\circ}$ e 25,3 de latitude Sul. Campos de Jordão, localizado na Serra da Mantiqueira, é o município paulista com sede situada em maior altitude (1.815 metros acima do nível do mar) e com temperaturas mais baixas (faixa de temperatura média de julho abaixo de $13^{\circ} \mathrm{C}$ ). Até 1995, não se verificou nesse município o estabelecimento de nenhuma das espécies estudadas. Todas as sedes dos demais municípios estão localizadas em áreas com isotermas de julho entre $13^{\circ} \mathrm{C}$ até pouco mais de $20^{\circ} \mathrm{C}$. Os índices pluviométricos variam de $1.200 \mathrm{~mm}$ até pouco mais de $3.000 \mathrm{~mm}$ anuais. ${ }^{4,16}$

A análise da influência da temperatura no processo de expansão geográfica da população de $A e$. aegypti

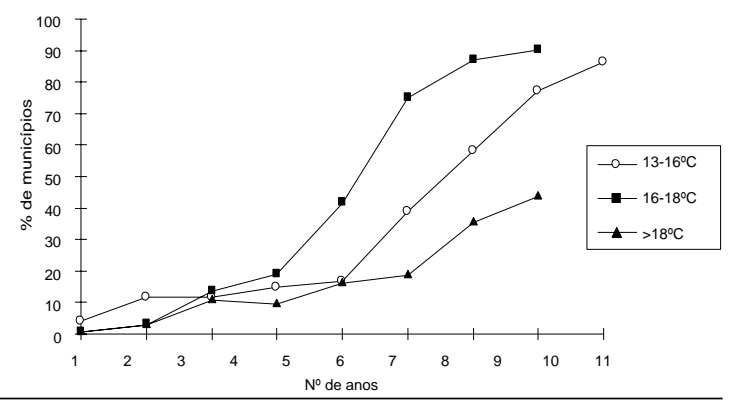

Figura 4 - Percentual de municípios infestados por Ae. albopictus em diferente áreas quanto à faixa de temperatura média de julho, segundo número de anos transcorridos após o estabelecimento da espécie em cada área. Estado de São Paulo. 1987 a 1995

Tabela 2 - Municípios com ocorrência de focos de Ae. albopictus, sem infestação anterior por Ae. aegypti, situados até 59 km de área infestada, segundo temperatura média do mês de julho e estabelecimento da espécie. Estado de São Paulo, $1987-$ 1995.

\begin{tabular}{|c|c|c|c|c|c|c|}
\hline \multirow[t]{2}{*}{$\begin{array}{l}\text { Temperatura } \\
\text { média }\end{array}$} & \multicolumn{2}{|c|}{$\begin{array}{c}\text { Municípios sem estabelecimento } \\
\text { da espécie }\end{array}$} & \multicolumn{2}{|c|}{$\begin{array}{c}\text { Municípios com estabelecimento } \\
\text { da espécie }\end{array}$} & \multicolumn{2}{|c|}{ Total } \\
\hline & $\mathrm{N}$ & $\%$ & $N$ & $\%$ & $\mathrm{~N}$ & $\%$ \\
\hline 13 a $16^{\circ} \mathrm{C}$ & 144 & 53,7 & 124 & 46,3 & 268 & 100,0 \\
\hline 16 a $18^{\circ} \mathrm{C}$ & 78 & 53,4 & 68 & 46,6 & 146 & 100,0 \\
\hline$>18^{\circ} \mathrm{C}$ & 10 & 35,7 & 18 & 64,3 & 28 & 100,0 \\
\hline Total & 232 & 52,5 & 210 & 47,5 & 442 & 100,0 \\
\hline
\end{tabular}

qui-quadrado $=3,38 ; p>0,05$ 
mostrou existência de três padrões distintos segundo faixa de temperatura média de julho. Esse fator parece atuar como modulador da expansão dessa espécie, uma vez que quanto mais baixa a temperatura, mais lento o processo. A temperatura parece preponderar sobre fatores como densidade demográfica e importância econômica das regiões, explicando por que regiões mais frias, como a da Grande São Paulo, Jundiaí, São José dos Campos, Taubaté e Sorocaba, mantiveram-se, até final de 1995, com poucos ou nenhum município infestado por Ae. aegypti. A velocidade intermediária da expansão geográfica da espécie em outras regiões de importância econômica e alta densidade demográfica, como Campinas, Piracicaba e Limeira, corrobora esse fato. Entretanto, o Litoral de São Paulo, apesar das altas temperaturas (acima de $18^{\circ} \mathrm{C}$ ou $16^{\circ} \mathrm{C} \mathrm{a} 18^{\circ} \mathrm{C}$ ), está entre as últimas regiões paulistas a apresentar infestação. ${ }^{6}$ Esse fato parece estar relacionado a seu distanciamento de áreas infestadas por Ae. aegypti no Estado de São Paulo e em estados vizinhos, pois quanto maior essa distância menos freqüente foi a ocorrência de focos dessa espécie, e menor foi o percentual de municípios onde se verificou seu estabelecimento. ${ }^{6}$ Além disso, a maioria dos focos detectados se restringiu ao Porto de Santos, onde as ações de vigilância e controle definidas pelo programa apresentam mais freqüência e intensidade por se tratar de porto internacional.

Para Ae. albopictus, cuja ampla distribuição geográfica no mundo inclui áreas com grande faixa de variação da temperatura, mostrando-se bem mais tolerante ao frio que Ae. aegypti, não se verificou no presente estudo uma relação clara dessa variável em seu processo de expansão geográfica. Não obstante a isto, foram detectados focos em quatro diferentes anos no município de Campos do Jordão, sem suceder seu estabelecimento, o que pode ter alguma relação com a elevada altitude do local. Em situação oposta, ou seja, em que a temperatura média de julho é superior a $18^{\circ} \mathrm{C}$, constatou-se menor velocidade de expansão geográfica da espécie. Questões como origem da população de Ae. albopictus do Brasil, se é de área tropical ou temperada com perda de resposta ao fotoperiodismo e de resistência ao frio ${ }^{9,15}$ não fizeram parte do presente estudo. No entanto, é interessante assinalar que, na macrorregião com faixa de temperatura de $13^{\circ} \mathrm{C} \mathrm{a} 16^{\circ} \mathrm{C}$, Ae. albopictus chegou a infestar $10 \%$ dos municípios dois anos após seu estabelecimento em algum município da área, enquanto Ae. aegypti infestou menos de $10 \%$ dos municípios após quatro anos, o que corrobora a maior capacidade de tolerância ao frio daquela espécie.

Com relação à influência dos índices pluviométricos no processo de expansão geográfica das duas espécies, não se verificou qualquer relação entre a variação desses índices e o estabelecimento de Ae. albopictus. Entretanto, para Ae. aegypti, apenas dentre os municípios pertencentes à área com temperatura média de julho na faixa de $16^{\circ} \mathrm{C}$ a $18^{\circ} \mathrm{C}$, verificou-se fraca associação entre essas variáveis, observando-se que quanto maiores os índices pluviométricos, maior o percentual de estabelecimento da espécie.

A redução de abundância das populações de $A e$. aegypti ou de Ae. albopictus, quando da sobreposição de sua distribuição, tem sido observada em diferentes regiões do mundo. Em Calcutá, Índia, na década de 30, e em várias cidades do Sudeste Asiático, nas décadas de 50 e 60, observou-se redução de abundância de $A e$. albopictus após a introdução de $A e$. aegypti. Segundo Chan et al, ${ }^{3}$ estudos por eles realizados e por vários outros autores foram inconclusivos, e consideraram provável que o padrão de distribuição desses culicídeos fosse resultante do favorecimento de Ae. aegypti pelo amplo e rápido processo de urbanização e pela maior fecundidade e menor ciclo de vida dessa espécie. No entanto, Ae. albopictus continuou a predominar em áreas rurais e periurbanas no Sudeste Asiático. ${ }^{3}$ Mais recentemente (décadas de 80 e 90), constatou-se redução da abundância de Ae aegypti em diversas cidades dos Estados Unidos após a introdução de Ae. albopictus. Vários experimentos vêm sendo realizados para avaliar esse fenômeno, porém os resultados encontrados não sugerem que a cepa de Ae. albopictus presente nesse país seja mais competitiva que a de Ae. aegypti. Outras explicações têm sido colocadas, como o fato do pneu ser o principal ecótopo para as duas espécies e ser freqüentemente encontrado no extradomicílio, o que poderia favorecer Ae. albopictus; além disso, existe a possibilidade de a redução de abundância de $A e$. aegypti ocorrer independentemente de $A e$. albopictus, pois oscilações na abundância daquela espécie já ocorreram nesse país, principalmente nas décadas de 50 e $60 . .^{1,10,15}$

Em 1986, um ano depois da detecção de municípios infestados por Ae. aegypti a Oeste do Estado de São Paulo, ${ }^{6}$ foi registrada a introdução de $A e$. albopictus em vários municípios do Vale do Paraíba, ${ }^{5}$ sendo que os primeiros municípios infestados pelas duas espécies surgiram em 1989 ao Norte do Estado. ${ }^{6}$ No processo de expansão geográfica desses culicídeos, não se encontrou associação entre infestação anterior por Ae. albopictus e estabelecimento de Ae. aegypti. Entretanto, a existência de formas diferentes de conduta para controle de $A e$. albopictus, uma para municípios já infestados por Ae.aegypti e outra para aqueles não infestados, ${ }^{6}$ pre- 
judicou o estudo de associação entre infestação anterior por Ae. aegypti e estabelecimento de $A e$. albopictus. Estudos incluindo avaliação dos níveis de densidade larvária e outros indicadores de abundância dessas espécies nas várias regiões paulistas, em áreas rurais, periurbanas e urbanas e nos ambientes intra e extradomiciliares, bem como a identificação dos ecótopos preferenciais, serão de grande importância para aprofundar o conhecimento sobre o padrão de distribuição das populações desses culicídeos no Estado de São Paulo e sobre a existência de possível competição entre as mesmas.

\section{AGRADECIMENTOS}

Aos técnicos da Seção de Climatologia Agrícola do Instituto Agronômico de São Paulo, SP, e especialmente ao Dr. Marcelo B. Paes de Camargo, pelo fornecimento de dados meteorológicos e orientações técnicas sobre as cartas climáticas.

\section{REFERÊNCIAS}

1. Black WC, Rai KS, Turco BJ, Arroyo DC. Laboratory study of competition between United States strains of Aedes albopictus and Aedes aegypti (Diptera: Culicidae). J Med Entomol 1989;26:260-71.

2. Berquó ES, Souza JMP, Gotlieb SLD. Análise descritiva de uma distribuição de freqüências a duas variáveis qualitativas. In: Berquó ES, Souza JMP, Gotlieb SLD. Bioestatística. São Paulo: EPU; 1981. p. 47-61.

3. Chan KL, Chan YC, Ho BC. Aedes aegypti (L) and Aedes albopictus (Skuse) in Singapore City 4. Competition between species. Bull World Health Organ 1971;44:643-9.

4. Departamento de Águas e Energia Elétrica. Totais mensais de chuva do Estado de São Paulo. São Paulo: Secretaria de Recursos Hídricos; 1993.

5. Forattini OP. Identificação de Aedes (Stegomyia) albopictus no Brasil. Rev Saúde Pública 1986;20:244-5.

6. Glasser CM, Gomes AC. Infestação do Estado de São Paulo por Aedes aegypti e por Aedes albopictus. Rev Saúde Pública 2001;34:570-7.

7. Githeko AK, Lindsay EW, Confalonieri EU, Patz JA. Climate change and vector-borne diseases: a regional analysis. Bull World Health Organ 2000;78:1136-47.

8. Gubler DJ. Dengue. In: Monath TP. The arboviruses epidemiology and ecology. Boca Raton, Florida: CRC Press; 1988. v. 2 p. 223-60.
9. Hanson SM, Craig JRGB. Cold acclimation, diapause, and geographic origin affect cold hardiness in eggs of Aedes albopictus (Diptera: Culicidae). I Med Entomol 1994;31:192-201.

10. Ho BC, Ewert A, Chew L. Interspecific competition among Ae. aegypti, Ae. albopictus and Ae. triseriatus (Diptera: Culicidae): larval development in miscid cultures. I Med Entomol 1989;26:615-23.

11. Knudsen AB, Slooff R. Vector-borne disease problems in rapid urbanization: new approaches to vector control. Bull World Health Organ 1992;70:1-6.

12. Nawrocki SJ, Hawley WA. Estimation of the northern limits of distribution of Aedes albopictus in North America. J Am Mosq Control Assoc 1987;3:314-7.

13. Nelson MJ, Suarez MF, Archila LF, Guzman, J. The distribution of Aedes aegypti (L) at high elevations in Colombia. Genva: WHO; 1983. (WHO/VBC 872)

14. Organización Panamericana de la Salud. Dengue y dengue hemorrágico en las Américas: guias para su prevencion y control. Washington (DC); 1995. (OPSPublicacion Científica, 548).

15. Rai KS. Aedes albopictus in the Americas. Annu Rev Entomol 1991;36:459-84

16. Secretaria de Estado da Agricultura. Zoneamento agrícola do Estado de São Paulo. São Paulo; 1974. v. 1. 